\title{
A New Nodal Stress Recovery Technique in Finite Element Method Using Colliding Bodies Optimization Algorithm
}

\author{
Ali Kaveh ${ }^{1 *}$, Mohammad Reza Seddighian ${ }^{1}$ \\ ${ }^{1}$ Department of Civil Engineering, Iran University of Science and Technology, Narmak, Postal Code 1684613114, Tehran, Iran \\ ${ }^{*}$ Corresponding author, e-mail: alikaveh@iust.ac.ir
}

Received: 06 August 2019, Accepted: 11 October 2019, Published online: 28 November 2019

\begin{abstract}
In Finite Element Method (FEM), the stress components are calculated within the elements firstly, and then these components are recovered to the nodes. For the recovery process, there are several well-known methods in which the increase of their accuracy imposes additional costs into the problem. In this paper, a new nodal stress recovery technique is proposed in which Colliding Bodies Optimization ( $\mathrm{CBO}$ ) Algorithm fits an appropriative function for nodal stress fields. The CBO employs this function to compute the stress components in the nodal coordinates. Therefore, a particular model to stress fields and its components will be available. It can be considered as a connection between analytical approaches and numerical methods, providing benefits of both categories. Finally, the accuracy, efficiency, and applicability of the new technique are investigated employing three diverse examples.
\end{abstract}

Keywords

Finite Element Method, error estimation, optimization, metaheuristics, Colliding Bodies Optimization

\section{Introduction}

In Finite Element Method (FEM) part of the computation is accomplished within the elements. However, significant entities must report as objectives in the nodes. Hence, the data transfer from the elements to the nodes is required. This procedure is called Recovery, that is an important step of FEM. A node in FEM mesh is affected by several elements incident with that node, so-called as Reference Node. These set of nodes and elements are known as a patch, Fig. 1(a), (b).

The initial idea for the nodal stress recovery is to average the stress values within a patch and consider the results as stress components of the reference node. This procedure which is called Averaging is the simplest and the least accurate technique to achieve the nodal stress components. In usual cases, in which the behavior of stress fields in a patch is almost uniform, there is no obstacle to estimate the stress components of a reference node in this manner. In the mentioned conditions, the averaging technique may propound suitable solutions. On the other hand, in problems which suffer from turbulent stress fields, it is not effortless to approximate the nodal stress components by averaging. This difficulty often leads to a considerable decrease in the accuracy of the solutions influencing the correctness of the

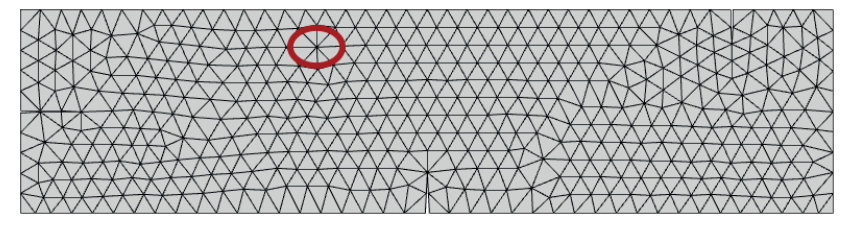

(a)

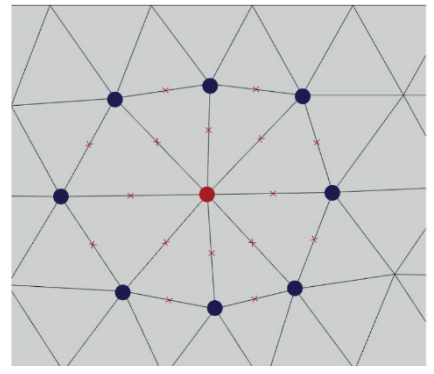

(b)

Fig. 1 (a) A general FEM mesh and a patch. (b) The related patch of a reference node (shown in red)

final results. In addition to this problem, when a numerical method is employed to compute the solutions, availability of an appropriate analytical function and/or field which can model the behavior of a node in a specific patch is usually an unattainable ambition. Accordingly, the stress recovery is considered as an important part in numerical analysis. 
To validate the final results of any stress recovery technique, it is necessary to find out how much the solutions are accurate. This procedure is usually called error estimation. Since there is no exact solution in the problems by which the relative error can be calculated, a criterion must define to accomplish this errand.

Generally, there are two classes of techniques to error estimation, which are divided into the Residual Based and the Recovery Based methods. Originally, the residual based technique is introduced by Babuška and Rheinboldt [1] that presumes the local residuals of solutions. The employed error estimator in this method is based on the norm of energy that is computed by residuals in a patch of elements. In the following, this technique is continued by several researchers as Ainsworth and Oden [2]. Then again, another error estimator method suggested based on executing the recovery process to improve the accuracy of numerical solutions by Zienkiewicz and Zhu [3]. In this method, the approximation of error is defined as the difference between the recovered and numerical solutions. They also proved that if the recovery technique will be Superconvergent, then the estimator is always asymptotically exact $[4,5]$. One of the most important studies is carried out by Zhu and Zhang [6]. They proved that for each residual based estimator, there is a corresponding recovery based process. Given that the same thing cannot be said for the opposite situation, the recovery based methods with optimal performance does not seem to have an alternative residual process. Therefore, the scope of applications of recovery based methods is greater.

As mentioned, in the recovery based procedure, the approximation of error is defined as the difference between the recovered and numerical solutions. Hence, the existence of a method to obtain recovered solutions is obligatory. The most straightforward approach for solution recovery, as mentioned previously, is to average the stress values within the patch (Averaging Technique). Zienkiewicz and Zhu [4, 7] presented the Superconvergent Patch Recovery (SPR) technique, one of the most effective proposed methods for recovery of solutions. Since the gradients of the finite element approximation exhibit superconvergence at the Gauss integration points of isoparametric elements [8-11], SPR technique will lead to more accurate solutions. Moslemi and Khoei [5] developed Weighted SPR by adding a parameter indicates the distance of a patch point from a reference node into the SPR formulation. Khoei et al. [12] proposed the Modified Superconvergent Patch Recovery (MSPR) technique by further improvements on the formulations of the SPR.
Anitescu et al. [13] executed a recovery based error estimation technique in high-order splines. For the low order 3D FEM, Sharma et al. [14] improved stress recovery. Wang et al. [15] employed superconvergence analysis for solving Maxwell's equations and Yuan et al. [16] developed a new adaptive finite element method (AFEM) based on element energy projection method. This field advanced by efforts of Patton et al. [17] on laminated composite plastic and endeavors of Chi et al. [18]. Caldern et al. [19], Kim and Jang [20], Sracic and Elke [21], Boffi and Gastaldi [22], Prato Torres et al. [23], Ganis et al. [24] and Song et al. [25] advanced this field by essays and all of them performed efforts on applications of error estimation and the AFEM.

For employing mentioned methods, the number of patch points must be equal to the unknowns, i.e., when the C1SPR in which a quadratic surface is fitted to the patch is employed, the corresponding data of at least six points are required based on Khayyam ${ }^{1}$ - Pascal's triangle. It means that at least six points must be available in the related patch. By increasing the necessity of more accuracy in the fitted function and/or model, the number of compulsive nodes also will increase, and this informs that the problem must solve by a more powerful element. This issue finally leads to an impressive increment of the procedure cost. The second limitation of executing the prevalent methods which will be called in the following as Classic Methods is the type of selected functions. In classic methods, the formulations are based on polynomial functions, i.e., only finite sentences of polynomials can be selected in the solving procedure. Furthermore, since the number of points in a related patch of a node is finite, therefore the selection of high order powers in polynomial function is almost impossible. Hence, this note informs about the third limitation of classic methods. These three limitations are not all the drawbacks of classic methods. When the area of an element decrease (the phenomenon occurs in common problems and exactly is an aim of adaptive finite element method procedure), the coordinates of points in a patch will be very close together. This property leads to a singularity in the formulation of the classic methods. Therefore, for many classes of problems, it is not feasible for this category of techniques to estimate an appropriate model of variables behavior.

In this paper, a new method is proposed to recover the stress components from a patch to a reference node using a metaheuristic algorithm. For this objective, the Colliding Bodies Optimization (CBO) Algorithm is employed.

1 Ghíyath al-Din Abu'l-Fath Umar ibn Ibrahim Al-Nisaburi al-Khayyam 
Furthermore, an exclusive model for each stress component is fitted based on the particular behavior of each node. In the following, the accuracy of the proposed method is evaluated in the first example. In the second and third examples, two types of FEM generally problems, i.e., crack propagation and mesh adaptivity are solved in order to be assured that the proposed method can successfully take the place of classic methods in the Stress Recovery problems and their applications.

\section{Adaptive Finite Element Method}

An auxiliary approach for improving the accuracy of the FEM is the Adaptive Finite Element Method (AFEM) which generally is divided into three categories H-Adaptivity, P-Adaptivity, and R-Adaptivity [26, 27]. According to this approach, the initial mesh will be updated based on error estimator commands, and by this trick, the total error over the problem domain will decrease. Figs. 2 to 5 indicate the

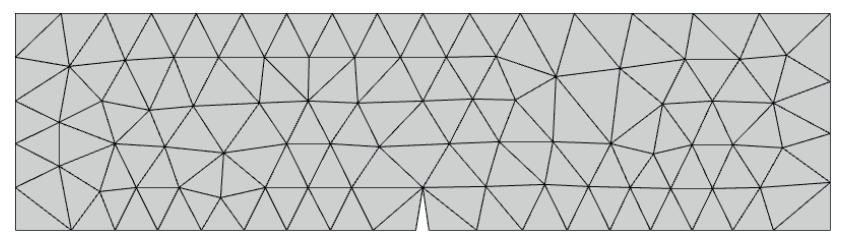

Fig. 2 The simple beam with a central notch and its initial mesh

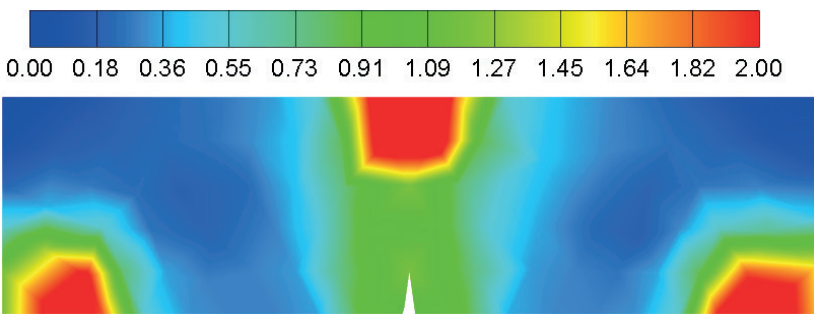

Fig. 3 The stress error contour of Fig. 2 (based on the SPR technique)

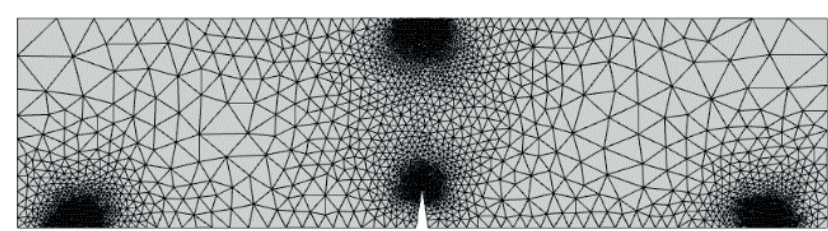

Fig. 4 The updated mesh of Fig. 2 Based on AFEM

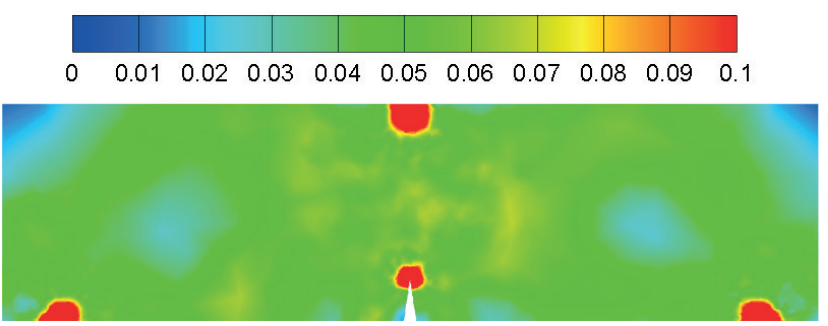

Fig. 5 The stress error contour of Fig. 4 (based on the SPR technique) initial and the final meshes of a simple beam with a central notch. As shown in Fig. 3, the stress error over the problem domain in the most zones is more than 60 percent and in the critical nodes same as restrained nodes in which there is the stress intensity this error is more than 200 percent. By employing an AFEM procedure initial mesh (Fig. 2) updated to an appropriate mesh (Fig. 4) in which the stress error over the domain in most zones is less than 5 percent, and there are high error values only in finite nodes containing stress singularity in their proximity (Fig. 5).

The AFEM is employed widespread in various fields of science including engineering, mathematics, physics, etc. Moslemi and Tavakkoli [28] could develop a static approach for error estimation in AFEM. Khoei et al. [29] modeled cohesive crack propagation using AFEM and advanced this issue [30] by modeling of non- planar cohesive fracture crack growth via AFEM. Also, Moslemi and Khoei [31] employed AFEM for modeling of damage growth and crack initiation. The applications of AFEM are not confined to inducted instances. This subject includes many other fields too. Bahbah et al. [32] employed AFEM in an anisotropic framework. de-Pouplana and Oñate [33] could combine this field with non-local damage for quasi-brittle materials. For compressible flows, Guaily and Megahed [34] executed AFEM for planar and axisymmetric problems. Electromagnetics is another field in which AFEM successfully is employed. This procedure is released by Ledger et al. [35] by developing an HP-Adaptivity method for scattering problems. The isogeometric analysis also influenced by the adaptivity procedure. Thermomechanics also is a field of science which uses adaptivity benefits. Badnava et al. [36] released an H-Adaptivity phase-field model for fractures in this concept. Soghrati et al. [37] developed an automated model-based on the AFEM for microstructures. Adaptive FEM is employed for RC shells by Lackner and Mang [38]. Lackner and Mang [39] released a paper for application of AFEM in concrete structures. Finally, there is a review essay on the adaptive techniques in the finite element method released by Chen [40].

Although, the AFEM is a helpful approach to decrease the error of numerical analysis, however, it is an iterative approach in which the problem must solve several times. Obviously, this property imposes an impressive cost on solving procedure. The other objectionable note for AFEM is that since the AFEM executes stress recovery techniques for achieving its final results, all the three drawbacks which mentioned for classic methods also exist for it. 


\section{Colliding Bodies Optimization algorithm}

The classic methods for finding solving procedures and efficient remedies in Structural Mechanics problems are finite. Also, the proposed procedures by them require complementary data, as mentioned in Section 1. One of the appropriate alternative methods instead of the problem solving procedure is Artificial Intelligence (AI). In the scope of this paper, Artificial Intelligence can employ for:

A. Recovering the stresses from a patch to a specified node.

B. Fitting an appropriative stress function based on the exclusive behavior of the patch node.

The AI methods can solve broad fields of problems without mentioned limitations for classic methods. One of the most popular and efficient implements of AI methods are Metaheuristics Algorithms. Metaheuristics Algorithms start problem-solving from several points, and if the employed method is commensurate to problem conditions, it can solve problem rapidly and more accurate and comfortable. These benefits are alongside two main traits of them namely the self- governing state from complementary data, and they limitation less property.

Nowadays, the metaheuristics are employed widely in various fields of science and are executed for several objectives same as optimization, modeling, etc. This Mega-Trend also contains Structural Engineering. Kaveh et al. [41] applied the ant colony algorithm on the optimization of the topology of structures. Kaveh and Talatahari [42] designed the skeletal structures using the imperialist competitive algorithm. Rahami et al. [43] combined force method and genetic algorithm to optimize size, geometry, and topology of trusses. Kaveh and Zolghadr [44] employed the magnetic charged system search for structural optimization. Lebaal [45] developed a robust model optimizer based on metaheuristic approaches. Powder compaction design procedure is one of the most important and famous problems in computational mechanics. In this filed, also the metaheuristics are applied, and this trick leads to relevant results. New efforts and advances in metaheuristics and their applications can be found in Kaveh [46, 47].

Generally, nature attempts to perform optimally. It achieves this by a trial and error procedure and spending time as the cost. Since the physical rules describe nature treatment, therefore, the laws of physics can be considered as an optimizer. The employing of their approach can be useful for solving complicated problems. This fact is the base of many metaheuristics algorithms same as
Genetic Algorithm (GA), Particle Swarm Optimization (PSO), Ant Colony Optimization (ACO), Colliding Bodies Optimization (CBO) and many other instances.

The CBO Algorithm is developed by Kaveh and Mahdavi [48] in 2014. The base of this optimization algorithm is the collisions between two bodies which are governed by the law of momentum and energy. If the studied system is isolated, then the total momentum of the system will also be conserved. This property leads to equality of momentum of all objects before and after the collision if there is no net external force acting upon the objects. These details can be expressed by:

$m_{1} v_{1}+m_{2} v_{2}=m_{1} v_{1}^{\prime}+m_{2} v_{2}^{\prime}$,

where $v_{1}$ and $v_{2}$ are the initial velocities of the first and the second bodies before the collision, $v_{1}^{\prime}$ and $v_{2}^{\prime}$ are the final velocities of the first and the second bodies after collisions and, $m_{1}, m_{2}$ are the mass of two bodies, respectively.

According to the conservation of the total kinetic energy the Eq. (2) can be expressed.

$\frac{1}{2} m_{1} v_{1}^{2}+\frac{1}{2} m_{2} v_{2}^{2}=\frac{1}{2} m_{1} v_{1}^{\prime 2}+\frac{1}{2} m_{2} v_{2}^{\prime 2}+Q$

where $Q$ is the loss of kinetic energy during the collision (Fig. 6).

In a one dimensional collision, the final velocities can be calculated using Eqs. (3) and (4).

$v_{1}^{\prime}=\frac{\left(m_{1}-\xi m_{2}\right) v_{1}+\left(m_{2}+\xi m_{2}\right) v_{2}}{m_{1}+m_{2}}$

$v_{2}^{\prime}=\frac{\left(m_{2}-\xi m_{1}\right) v_{2}+\left(m_{1}+\xi m_{1}\right) v_{1}}{m_{1}+m_{2}}$

where $\xi$ is the Coefficient of Restitution (COR) of the two colliding bodies defined as Eq. (5).

(A)

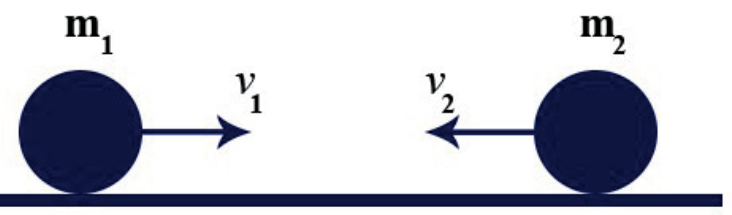

(B)

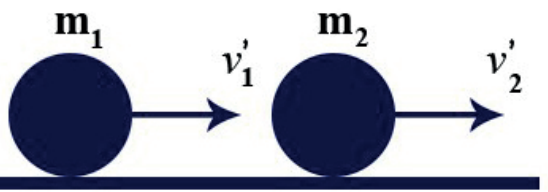

Fig. 6 The collision between two bodies. (A) Before the Collision and (B) after the collision 
$\xi=\frac{\left|v_{2}^{\prime}-v_{1}^{\prime}\right|}{\left|v_{2}-v_{1}\right|}$

In Colliding Bodies Optimization (CBO) Algorithm, each solution contains some variables which are considered as a Colliding Body (CB). There are two categories of objects which are called Massed Objects. Mass objects are divided into Stationary Objects and Moving Objects. As a result of objects motions, the collisions occur between pairs of objects. These collisions lead to (i) improve the positions of moving objects and (ii) to push stationary objects toward more appropriate positions. After the collision, the positions, the velocities and the masses of bodies will be updated based on new conditions. This procedure will repeat until the appropriate solution will be achieved. The CBO algorithm is employed for widespread structural engineering problems. Kaveh and Ardalani [49] optimized the cost and $\mathrm{CO} 2$ emission of reinforced concrete frames using CBO. Optimal nonlinear analysis and design of double-layer grids are advanced by Kaveh and Moradveisi [50] also using CBO. Optimal large-scale space steel frames [51], structural optimization of jacket supporting structures for offshore wind turbines [52] and continuous and discrete optimization for design [53] also are problems which are solved using CBO. There are MATLAB codes, outlines, and examples of $\mathrm{CBO}$ in [54] for enthusiasts.

\section{Smart nodal stress recovery}

The main objective of this study, as described in Section 1, is to present an accurate, more comfortable, and more importantly, an efficient method to recover stress components from a patch into a reference node. The Proposing method must be limitless, which can apply for any problem in front of mentioned properties. For this objective, the modeling of an appropriate function for each node proprietary behavior based on CBO algorithm is employed. If the fitted function can be modeled in addition to recover the nodal stress components, an appropriate primarily function for each node is calculated as well and this is a connection between analytical and numerical approaches, i.e., the researcher by using this procedure can utilize both analytical and numerical methods benefits.

According to the Taylor series, it is possible to approximate the behavior of a function by a series of polynomials as presented in Eq. (6) for a one dimensional function.

$$
f(x)=a_{0}+a_{1} x+a_{2} x^{2}+a_{3} x^{3}+\ldots
$$

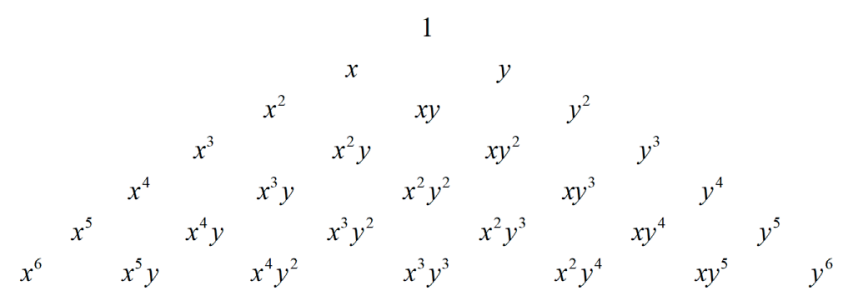

Fig. 7 The terms of Khayyam- Pascal's triangle

If the function is defined in two- dimensional coordinates, the Eq. (7) can be approximated for it and its terms achieve from Khayyam-Pascal's triangle ${ }^{2}$ (Fig. 7).

$$
z=f(x, y)=a_{00}+a_{10} x+a_{01} y+a_{20} x^{2}+a_{11} x y+. .
$$

It is possible to define each stress components of nodes as a function of node coordinates. This function is similar to what defined as Eq. (7). The most important note is that there is no limitation to select the number of terms or maximum power. The authors believe that hitherto this benefit there is not in any other methods which presented for this objective. The last barrier in finding suitable function is to calculate coefficients. The CBO duty is precisely this procedure. $\mathrm{CBO}$ searches and finds the best coefficients based on each node behavior. Since the patch coordinates and their available stress values must satisfy fitted function, therefore, this information, i.e., Gauss points coordinates, and their calculated stress value from FEM can be used to balance coefficients in order to decrease the error of the fitted function as much as possible. The CBO is employed based on following Cost Function.

$E=\sum_{i=1}^{n}\left(f\left(x_{i}, y_{i}\right)-\sigma_{i}\right)$,

where $n$ is the number of points in the considered patch, $\sigma$ is the calculated stress by FEM, $f(x, y)$ is the result of the fitted function or recovered stress and $E$ is the total error between fitted function and calculated stresses over the patch or Cost Function.

2 The pattern of numbers which is known as Pascal's triangle, has been discovered before his time. The main formula of Pascal's triangle is due to an Indian mathematician Acharya Pingala. At around 953-1029, the Persian mathematician Al-Karaji published a book containing the first description of Pascal's triangle. It was later repeated by another Persian poet-astronomer-mathematician Umar Khayyam. Also, this triangle has been utilized in other fields at around the same time. Jia Xia (1010-1070) and Yang Hui (1238-1298) in China, Petrus Apianus (1495-1552) and Michael Stifel in Germany and some other mathematician are among the scientists who discover this triangle and developed its properties almost independently. 
The coefficients are managed by decreasing cost function due to $\mathrm{CBO}$ procedures. The computations will be finished if the cost function is equal to zero or very close to it.

After the described procedure, an appropriate function for each stress components (for example, in-plane problems $\sigma_{x}, \sigma_{y}$ and $\tau_{x y}$ ) is provided in which the proprietary behavior of each node is modeled. Therefore, by substituting the coordinates of the reference node the recovered values of its stress components can be calculated. So, both objective of this study is achieved. The efficiency of the proposed method will be more tangible by studying three varied examples presented in Section 5.

\section{Numerical examples}

The intelligent nodal stress recovery technique is introduced in previous sections. In order to evaluate the proposed algorithm, two vivificates must be done. The first is the survey of accuracy and the second is the applicability. For these objectives, three examples are designed which the first focuses on first aim and two others investigate the second purpose.

\subsection{Calculation of stress intensity factor}

In order to survey the accuracy of the proposed algorithm, it is logical to compare its results with the outcomes of classic methods. As mentioned in Section 1 the classic methods are not exact and generally are based on approximation. Therefore, although the results comparing two categories is a common approach, however, there is a better way for examination. If a problem that can be solved either by an analytical method or a numerical approach is selected, then comparing results will be more and more logical. Also, this approach can verify the accuracy of the proposed method more apparent and appropriate.

One of the essential parameters in Fracture Mechanics is the Stress Intensity Factor (SIF) which is represented in opening mode problems (mode I) by $K_{I}$. In some problems, this parameter can be calculated either by analytical or by numerical methods. For a simple beam with a central notch, as shown in Fig. 8, $K_{I}$ is calculated using Eq. (9).

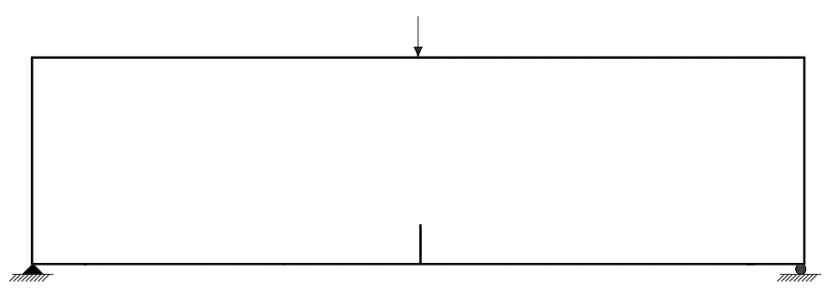

Fig. 8 Simple beam with the central notch

$$
\begin{aligned}
& K_{I}=\frac{P S}{B W^{3 / 2}}\left[2.9\left(\frac{a}{W}\right)^{1 / 2}-4.6\left(\frac{a}{W}\right)^{3 / 2}+21.8\left(\frac{a}{W}\right)^{5 / 2}\right. \\
& \left.-37.6\left(\frac{a}{W}\right)^{7 / 2}+38.7\left(\frac{a}{W}\right)^{9 / 2}\right],
\end{aligned}
$$

where $P$ is a concentrated load, $S, W, B$ are length, height, and thickness of the beam respectively. Here, $a$ is the length of the notch or initial crack. Details of Eq. (9) can be found in Fracture Mechanics References.

The SIF of the problem can be calculated analytically by substituting the described parameters into Eq. (9). For calculating the SIF by a numerical approach the following procedure can be utilized.

For static loading, the strain energy can be calculated by

$U=\frac{1}{2} P V$,

where $P, V$ and $U$ represent the concentrated load, relative vertical displacement, and the strain energy, respectively. If a crack propagates with a length of $\delta a$ and the difference between strain energy in initial and final states is equal to $\Delta U$, then the Strain Energy Release Rate can be obtained as

$G=\left|\frac{\Delta U}{\delta a}\right|$.

Finally, the SIF is calculated by

$K_{I}=\sqrt{E G}$,

where $E$ is the elasticity modulus.

In the described procedure, all the variables are available except the vertical displacement. This unknown can be calculated by FEM. Now SIF can be obtained by a numerical approach and then compared with the SIF calculated from the analytical method.

In order to find out the accuracy of the proposed algorithm in a simple beam with initial notch having a length of $4 \mathrm{~mm}$, the crack is propagated until a length $15 \mathrm{~mm}$ in 10 steps. The details of each step are shown in Table 1.

In each step, in the beginning, the related SIF is calculated by the analytical approach. Once the mesh is solved by FEM, the nodal stresses are calculated by the proposed algorithm. After that the mesh is updated based on the recovered stresses. After that, the problem is solved again based on the new mesh. The vertical displacement of concentrated load point is substituted in numerical formulation, and the corresponding SIF is computed. The final results of each step are compared and illustrated in Fig. 9. 
Table 1 The details of each step of Example 1

\begin{tabular}{cccccccc}
\hline No. & $\begin{array}{c}\mathrm{a} \\
(\mathrm{mm})\end{array}$ & $\mathrm{a} / \mathrm{W}$ & $\begin{array}{c}\mathrm{P} \\
(\mathrm{kg} . \mathrm{f})\end{array}$ & $\begin{array}{c}\mathrm{B} \\
(\mathrm{cm})\end{array}$ & $\begin{array}{c}\mathrm{W} \\
(\mathrm{cm})\end{array}$ & $\begin{array}{c}\mathrm{S} \\
(\mathrm{cm})\end{array}$ & $\begin{array}{c}\mathrm{E} \\
(\mathrm{kg} . \mathrm{f} / \mathrm{cm})\end{array}$ \\
\hline 1 & 4 & 0.07 & 40000 & 5 & 60 & 240 & 200000 \\
2 & 5.22 & 0.09 & 40000 & 5 & 60 & 240 & 200000 \\
3 & 6.44 & 0.11 & 40000 & 5 & 60 & 240 & 200000 \\
4 & 7.67 & 0.13 & 40000 & 5 & 60 & 240 & 200000 \\
5 & 8.89 & 0.15 & 40000 & 5 & 60 & 240 & 200000 \\
6 & 10.11 & 0.17 & 40000 & 5 & 60 & 240 & 200000 \\
7 & 11.33 & 0.19 & 40000 & 5 & 60 & 240 & 200000 \\
8 & 12.56 & 0.21 & 40000 & 5 & 60 & 240 & 200000 \\
9 & 13.78 & 0.23 & 40000 & 5 & 60 & 240 & 200000 \\
10 & 15 & 0.25 & 40000 & 5 & 60 & 240 & 200000 \\
\hline
\end{tabular}

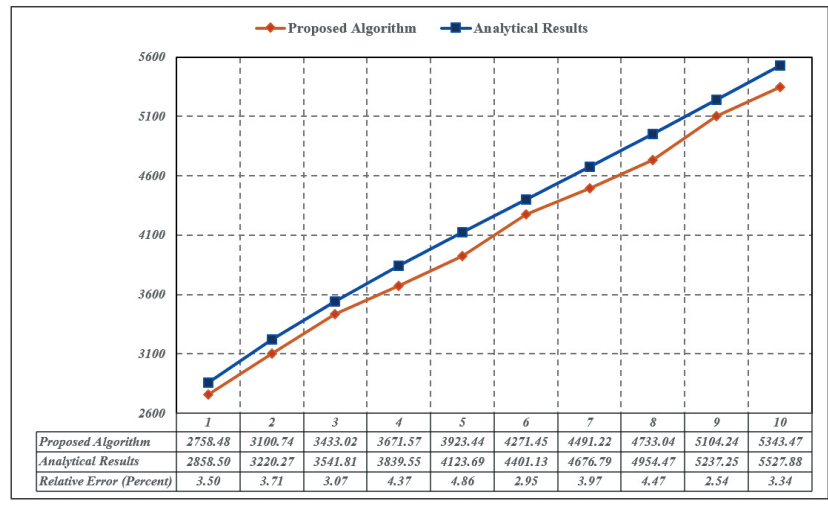

Fig. 9 The comparison between the results of the analytical and proposed algorithm

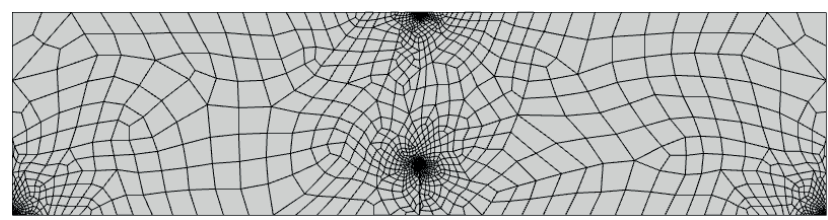

Fig. 10 The utilized mesh in FEM computation related to step 10

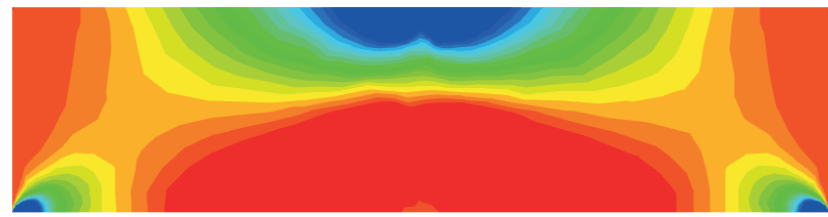

Fig. 11 The horizontal stress contour for step 10

The updated mesh for FEM computation and the horizontal stress $\sigma_{X}$ contour of step 10 are illustrated in Figs. 10 and 11 .

The duty of CBO, as mentioned in Section 4 is to fit an appropriate function for each node and each stress components of a node. This proper function for left restrained node step 10 illustrated in Fig. 12.
As illustrated in Fig. 12, CBO fitted a function based on patch points (shown in blue) and in the following computed the stress value of reference node, i.e. left restrained node based on its coordinates (Red point in Fig. 12). Since there is stress intensity in the support regions, the fitted function is got an infinite value in this region which verify the correction of modeling. This fitted function is in power 5 or utilized 21 terms of Khayyam-Pascal's triangle. As described in Section 3 there are no limits to select terms of Khayyam-Pascal's triangle and number of polynomials. The found coefficients of fitted function are presented in Table 2.

After employing the proposed method, an appropriate model for each node and each stress components are available, and this is the main achievement of this study. The second benefit of the proposed method is the accuracy of it in comparison with other classic methods. The procedure of decreasing the cost function in order to find the best solution by $\mathrm{CBO}$ is shown in Fig. 13.

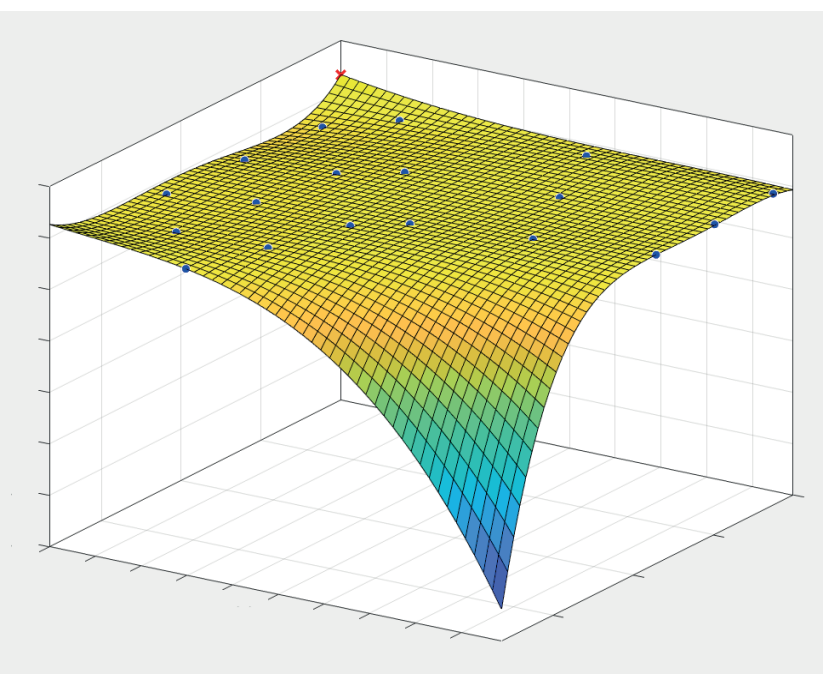

Fig. 12 The fitted function for the left restrained node of step 10

Table 2 The found coefficients by $\mathrm{CBO}$ for the fitted function of left restrained node in step 10 .

\begin{tabular}{cccccc}
\hline $\begin{array}{c}\text { Coeffi- } \\
\text { cient }\end{array}$ & Value & $\begin{array}{c}\text { Coeffi- } \\
\text { cient }\end{array}$ & Value & $\begin{array}{c}\text { Coeffi- } \\
\text { cient }\end{array}$ & Value \\
\hline $\mathrm{a}_{00}$ & $6.624 \mathrm{e}+05$ & $\mathrm{a}_{21}$ & $-1.303 \mathrm{e}+12$ & $\mathrm{a}_{04}$ & 0 \\
$\mathrm{a}_{10}$ & $-3.738 \mathrm{e}+08$ & $\mathrm{a}_{12}$ & $-1.465 \mathrm{e}+11$ & $\mathrm{a}_{50}$ & $-1.187 \mathrm{e}+14$ \\
$\mathrm{a}_{01}$ & $-3.099 \mathrm{e}+07$ & $\mathrm{a}_{03}$ & $-1.971 \mathrm{e}+09$ & $\mathrm{a}_{41}$ & $-5.002 \mathrm{e}+14$ \\
$\mathrm{a}_{20}$ & $4.043 \mathrm{e}+10$ & $\mathrm{a}_{40}$ & $2.803 \mathrm{e}+13$ & $\mathrm{a}_{32}$ & $-1.487 \mathrm{e}+14$ \\
$\mathrm{a}_{11}$ & $1.42 \mathrm{e}+10$ & $\mathrm{a}_{31}$ & $4.354 \mathrm{e}+13$ & $\mathrm{a}_{23}$ & $-2.306 \mathrm{e}+13$ \\
$\mathrm{a}_{02}$ & $4.26 \mathrm{e}+08$ & $\mathrm{a}_{22}$ & $9.394 \mathrm{e}+12$ & $\mathrm{a}_{14}$ & 0 \\
$\mathrm{a}_{03}$ & $-1.695 \mathrm{e}+12$ & $\mathrm{a}_{13}$ & $5.311 \mathrm{e}+11$ & $\mathrm{a}_{05}$ & 0 \\
\hline
\end{tabular}


As illustrated in Fig. 13, the initial population error is equal to 600 percent, and $\mathrm{CBO}$ could reduce this error or cost function to 0 by spending 1000 NFE. This computational power confirms that the proposed algorithm, alongside its mentioned benefits, is quite efficient and economical.

\subsection{Crack propagation problem}

The accuracy, efficiency, and outcomes of the proposed algorithm are studied in the first example. Another property of the proposed algorithm is its applicability to a wide range of numerical problems. In this example, in order to examine this property, a simple beam with non-central notch due to a concentrated load, and the crack route of propagation is investigated by the proposed algorithm, Fig. 14. Since the material is supposed to be perfectly brittle thus the crack propagation criterion is considered to be perpendicular to maximum principal stress. Therefore, the main variables to find the route of the crack propagation are crack tip stress components which are computed by the proposed algorithm. The logical and the correct found route indicate the credibility of the proposed algorithm.

The crack must propagate to the loaded node. This procedure is shown in Fig. 15.

As illustrated in Fig. 15, the route of crack propagation is following theories of fracture mechanics. This result informs that the recovered stress is credible.

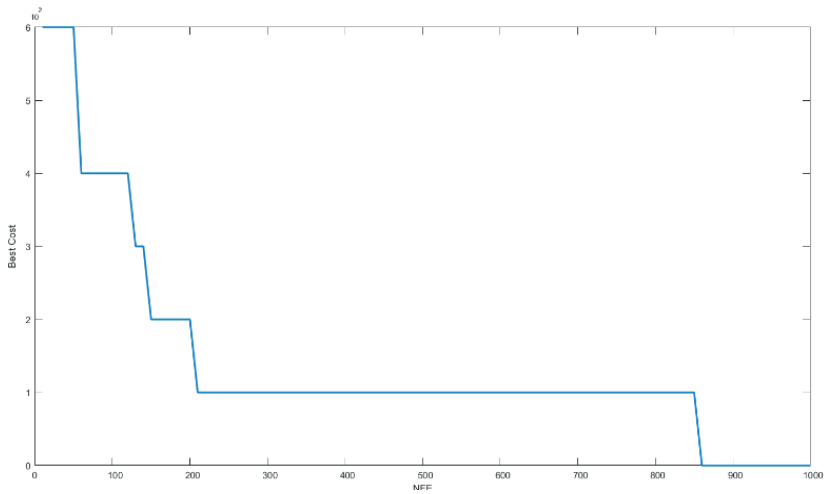

Fig. 13 The $\mathrm{CBO}$ process for finding the coefficients

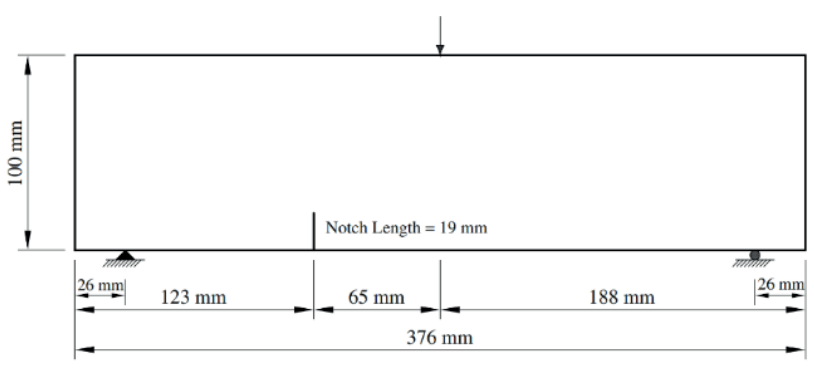

Fig. 14 The conditions of Example 2
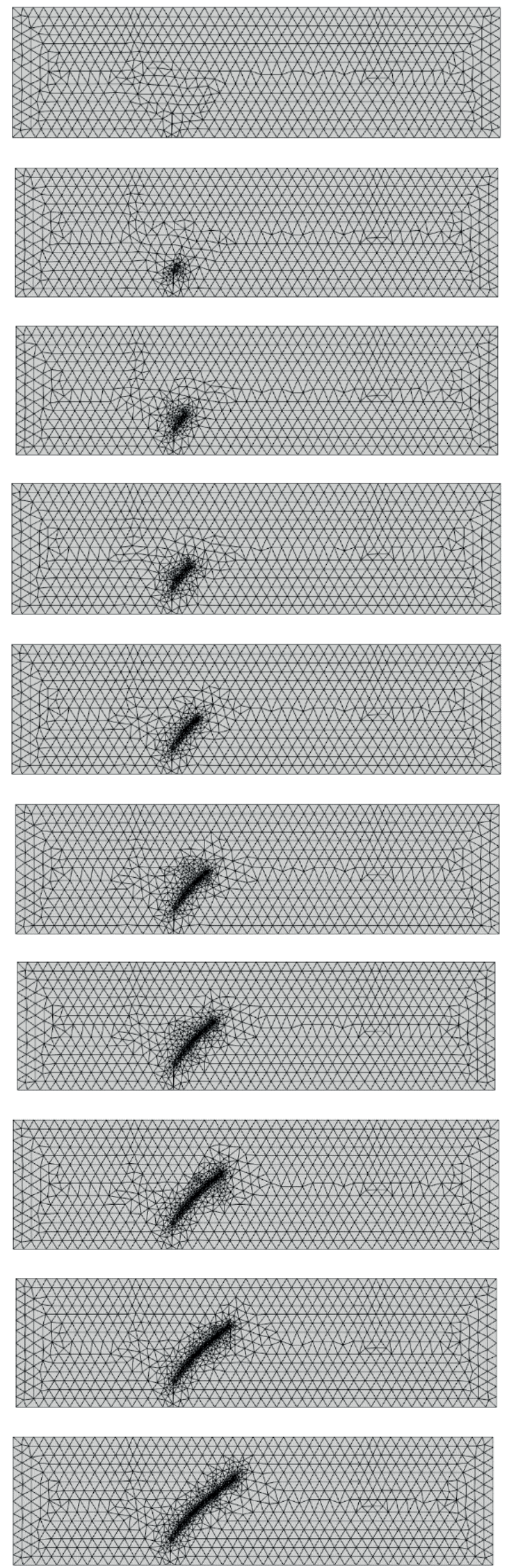

Fig. 15 The steps of the crack propagation 


\subsection{Error estimation and remeshing}

In order to verify the credibility of the proposed algorithm, another example is designed in which the mesh of a simple beam with three notches is updated by the proposed algorithm, Fig. 16. For adaptivity procedure one should initially calculate the error of analysis.

If assume $\sigma$ as the exact value of stress and $\hat{\sigma}$ as the value of stress calculated by finite element analysis, then it is possible to define the stress error as Eq. (13).

$e_{\sigma}=\sigma-\hat{\sigma}$

Since the exact solution for stress is not available, replacing improved stress $\sigma^{*}$ instead of it, is required.

$e_{\sigma} \simeq \sigma^{*}-\hat{\sigma}$

Due to some difficulties in practice, as the singularity of the stress field in the crack tip (i.e., the infinite value of error) the utilization of the norm of error is more appropriate. If the $L_{2}$ norm of the stress is defined as Eq. (15), then the Eq. (14) will convert to Eq. (16).

$$
\begin{aligned}
& \|e\|=\left(\int_{\Omega} e^{T} e d \Omega\right)^{1 / 2} \\
& \left\|e_{\sigma}\right\|=\left\|\sigma^{*}-\hat{\sigma}\right\|=\left(\int_{\Omega}\left(\sigma^{*}-\hat{\sigma}\right)^{T}\left(\sigma^{*}-\hat{\sigma}\right) d \Omega\right)^{\frac{1}{2}}
\end{aligned}
$$

Since generally analysis steps are computed in elements, the whole domain $\Omega$ reduces to each element domain, and the square of $L_{2}$ the norm of the overall domain will be obtained by summing element contributions as formulating in Eq. (17).

$\left\|e_{\sigma}\right\|^{2}=\sum_{i=1}^{m}\left\|e_{\sigma}\right\|_{i}^{2}$,

where $i$ represent element contribution and $m$ indicates the total number of elements. In this manner, the new mesh size is determined in such a way that produces uniform error distribution.

According to the described procedure, an initial mesh can be updated by evaluating errors and improving the

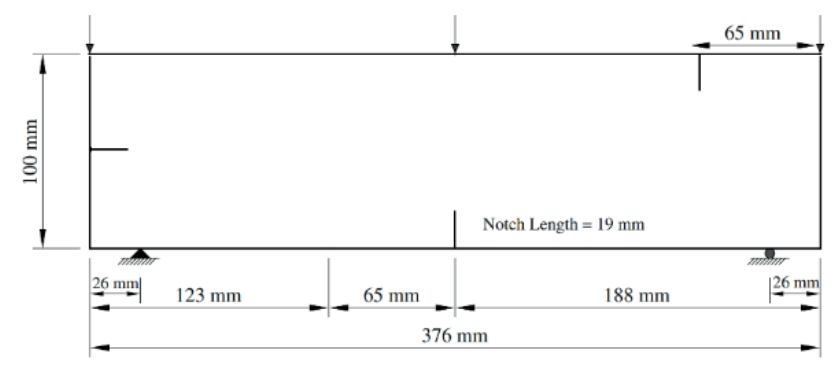

Fig. 16 The state of Example 3 mesh data based on errors, Fig. 17. This procedure is due to stress recovery, and the proposed algorithm is employed to this duty for a simple beam with three initial notches.

The final mesh which is shown in Fig. 18 is an appropriate mesh for FEM analysis with tiny error percent, Fig. 19. This example also indicates that the intelligent nodal stress recovery technique which is proposed in this study is an efficient, economical, accurate and practicable method for improving stresses from patches to nodes and has this potential to be employed in a wide range of finite element problems instead of using the classic methods.

\section{Conclusions}

In this paper, a new method is presented to recover the stress components from a patch to its corresponding reference node. The proposed method utilizes a metaheuristic algorithm to fit an appropriative nodal function which can calculate the nodal stress components for any problems. To evaluate the accuracy of the proposed method, a fracture mechanics benchmark problem as the first example is solved. The stress intensity factor is calculated using both the analytical and the proposed methods. Then, the final results are compared together, which the relative error in each step was less than five percent. Subsequently, in the second and third examples, the efficiency and applicability of the proposed method

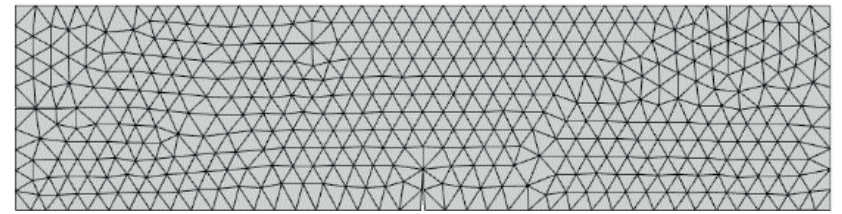

Fig. 17 The initial mesh of Example 3

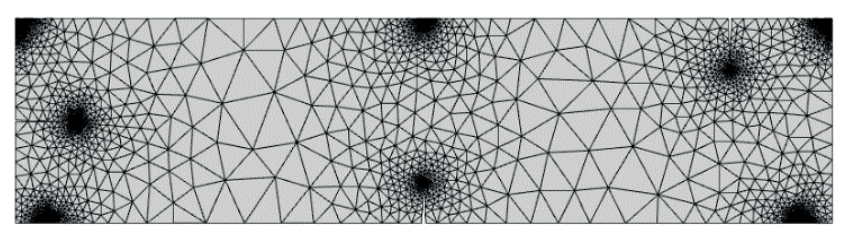

Fig. 18 The final mesh of Example 3 accomplished by the proposed algorithm

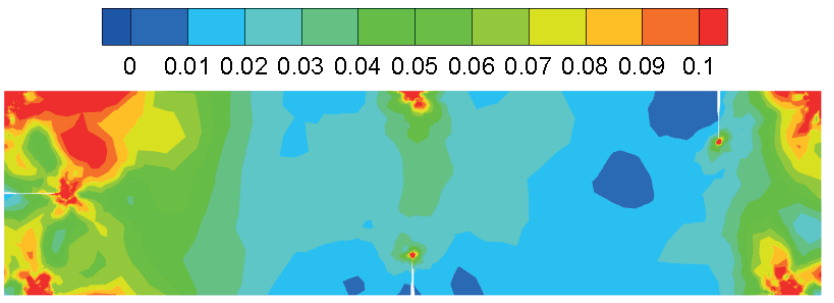

Fig. 19 The stress error contours 
are investigated. It is shown that the proposed method can take the place of the well-known methods same as averaging, SPR, etc., in the widespread FEM problems such as

\section{References}

[1] Babuška, I., Rheinboldt, W. C. "A posteriori error estimates for the finite element method", International Journal for Numerical Methods in Engineering, 12(10), pp. 1597-1615, 1978.

https://doi.org/10.1002/nme.1620121010

[2] Ainsworth, M., Oden, J. T. "A unified approach to a posteriori error estimation using element residual methods", Numerische Mathematik, 65(1), pp. 23-50, 1993. https://doi.org/10.1007/BF01385738

[3] Zienkiewicz, O. C., Zhu, J. Z. "A simple error estimator and adaptive procedure for practical engineering analysis", International Journal for Numerical Methods in Engineering, 24(2), pp. 337-357, 1987.

https://doi.org/10.1002/nme.1620240206

[4] Zienkiewicz, O. C., Zhu, J. Z. "The superconvergent patch recovery and a posteriori error estimates. Part 2: Error estimates and adaptivity", International Journal for Numerical Methods in Engineering, 33(7), pp. 1365-1382, 1992.

https://doi.org/10.1002/nme.1620330703

[5] Moslemi, H., Khoei, A. R. "3D adaptive finite element modeling of non-planar curved crack growth using the weighted superconvergent patch recovery method", Engineering Fracture Mechanics, 76(11), pp. 1703-1728, 2009.

https://doi.org/10.1016/j.engfracmech.2009.03.013

[6] Zhu, J. Z., Zhang, Z. "The relationship of some a posteriori error estimators", Studies in Applied Mechanics, 47, pp. 25-42, 1998. https://doi.org/10.1016/S0922-5382(98)80003-2

[7] Zienkiewicz, O. C., Zhu, J. Z. "The superconvergent patch recovery and a posteriori error estimates. Part 1: The recovery technique", International Journal for Numerical Methods in Engineering, 33(7), pp. 1331-1364, 1992.

https://doi.org/10.1002/nme.1620330702

[8] Chen, C. M. "Superconvergence of finite element solutions and their derivatives", Numerical Mathematics, a Journal of Chinese Universities, 3(2), pp. 118-125, 1981.

[9] Zienkiewicz, O. C., Taylor, R. L. "The finite element method, Vol. 2", Dynamics and non-linearity, McGraw-Hill, New York, NY, USA, 1991.

[10] Zlámal, M. "Superconvergence and Reduced Integration in the Finite Element Method", Mathematics of Computation, 32(143), pp. 663-685, 1978. https://doi.org/10.2307/2006479

[11] Mackinnon, R. J., Carey, G. F. "Superconvergent derivatives: A Taylor series analysis", International Journal for Numerical Methods in Engineering, 28(3), pp. 489-509, 1989.

https://doi.org/10.1002/nme.1620280302

[12] Khoei, A. R., Azadi, H., Moslemi, H. "Modeling of crack propagation via an automatic adaptive mesh refinement based on modified superconvergent patch recovery technique", Engineering Fracture Mechanics, 75(10), pp. 2921-2945, 2008. https://doi.org/10.1016/j.engfracmech.2008.01.006 crack propagation and remeshing. In both examples, the presented method led to acceptable results in an economical computational cost.

[13] Anitescu, C., Hossain, M. N., Rabczuk, T. "Recovery-based error estimation and adaptivity using high-order splines over hierarchical T-meshes", Computer Methods in Applied Mechanics and Engineering, 328, pp. 638-662, 2018.

https://doi.org/10.1016/j.cma.2017.08.032

[14] Sharma, R., Zhang, J., Langelaar, M., van Keulen, F., Aragón, A. M. "An improved stress recovery technique for low-order 3D finite elements", International Journal for Numerical Methods in Engineering, 114(1), pp. 88-103, 2018.

https://doi.org/10.1002/nme.5734

[15] Wang, L., Zhang, Q., Zhang, Z. "Superconvergence Analysis and PPR Recovery of Arbitrary Order Edge Elements for Maxwell's Equations", Journal of Scientific Computing, 78(2), pp. 1207 1230, 2019. https://doi.org/10.1007/s10915-018-0805-8

[16] Yuan, S., Dong, Y., Xing, Q., Fang, N. "Adaptive Finite Element Method of Lines with Local Mesh Refinement in Maximum Norm Based on Element Energy Projection Method", International Journal of Computational Methods, pp. 1950-1958, 2019. https://doi.org/10.1142/S0219876219500087

[17] Patton, A., Dufour, J. E., Antolin, P., Reali, A. "Fast and accurate elastic analysis of laminated composite plates via isogeometric collocation and an equilibrium-based stress recovery approach", Composite Structures, 225, Article number: 111026, 2019. https://doi.org/10.1016/j.compstruct.2019.111026

[18] Chi, H., da Veiga, L. B., Paulino, G. H. "A simple and effective gradient recovery scheme and a posteriori error estimator for the Virtual Element Method (VEM)", Computer Methods in Applied Mechanics and Engineering, 347, pp. 21-58, 2019. https://doi.org/10.1016/j.cma.2018.08.014

[19] Calderón, G., Carrillo, J. C., Villamizar, J., Torres, C., Castillo, J. "Adaptivity in mimetic difference schemes: one-dimensional transient problems", Journal of Physics: Conference Series, 1159, Article number: 012019, 2019. https://doi.org/10.1088/1742-6596/1159/1/012019

[20] Kim, Y., Jang, U. G. "Element Search Methods for Large Deformation Finite Element Simulation in Geotechnical Fields", KSCE Journal of Civil Engineering, 23(2), pp. 534-540, 2019. https://doi.org/10.1007/s12205-018-1368-9

[21] Sracic, M. W., Elke, W. J. "Effect of Boundary Conditions on Finite Element Submodeling", In: Kerschen, G. (ed) Nonlinear Dynamics, Volume 1, Conference Proceedings of the Society for Experimental Mechanics Series, Springer, Cham, Switzerland, 2019, pp. 163-170. https://doi.org/10.1007/978-3-319-74280-9_16

[22] Boffi, D., Gastaldi, L. "Adaptive Finite Element Method for the Maxwell Eigenvalue Problem", SIAM Journal on Numerical Analysis, 57(1), pp. 478-494, 2019. https://doi.org/10.1137/18M1179389 
[23] Prato Torres, R., Domínguez, C., Díaz, S. "An adaptive finite element method for a time-dependent Stokes problem", Numerical Methods for Partial Differential Equations, 35(1), pp. 325-348, 2019. https://doi.org/10.1002/num.22302

[24] Ganis, B., Pencheva, G., Wheeler, M. F. "Adaptive mesh refinement with an enhanced velocity mixed finite element method on semi-structured grids using a fully coupled solver", Computational Geosciences, 23(1), pp. 149-168, 2019. https://doi.org/10.1007/s10596-018-9789-6

[25] Song, J. H., Maier, M., Luskin, M. "Adaptive finite element simulations of waveguide configurations involving parallel 2D material sheets", Computer Methods in Applied Mechanics and Engineering, 351, pp. 20-34, 2019. https://doi.org/10.1016/j.cma.2019.03.039

[26] Logan, D. L. "A First Course in the Finite Element Method", Cengage Learning, Boston, MA, USA, 2016.

[27] Akin, J. E. "Finite element analysis with error estimators: An introduction to the FEM and adaptive error analysis for engineering students", Elsevier, Oxford, UK, 2005.

https://doi.org/10.1016/B978-075066722-7/50032-1

[28] Moslemi, H., Tavakkoli, A. "A Statistical Approach for Error Estimation in Adaptive Finite Element Method", International Journal for Computational Methods in Engineering Science and Mechanics, 19(6), pp. 440-450, 2019.

https://doi.org/10.1080/15502287.2018.1558424

[29] Khoei, A. R., Moslemi, H., Ardakany, K. M., Barani, O. R., Azadi, H. "Modeling of cohesive crack growth using an adaptive mesh refinement via the modified-SPR technique", International Journal of Fracture, 159(1), pp. 21-41, 2009 https://doi.org/10.1007/s10704-009-9380-1

[30] Khoei, A. R., Moslemi, H., Sharifi, M. "Three-dimensional cohesive fracture modeling of non-planar crack growth using adaptive FE technique", International Journal of Solids and Structures, 49(17), pp. 2334-2348, 2012.

https://doi.org/10.1016/j.ijsolstr.2012.04.036

[31] Moslemi, H., Khoei, A. R. "3D Modeling of Damage Growth and Crack Initiation Using Adaptive Finite Element Technique", Scientia Iranica Transaction A: Civil Engineering, 17(5), pp. 372-386, 2010.

[32] Bahbah, C., Mesri, Y., Hachem, E. "Interpolation with restrictions in an anisotropic adaptive finite element framework", Finite Elements in Analysis and Design, 142, pp. 30-41, 2018. https://doi.org/10.1016/j.finel.2017.11.011

[33] de-Pouplana, I., Oñate, E. "Combination of a non-local damage model for quasi-brittle materials with a mesh-adaptive finite element technique", Finite Elements in Analysis and Design, 112, pp. 26-39, 2016. https://doi.org/10.1016/j.finel.2015.12.011

[34] Guaily, A., Megahed, A. "An adaptive finite element method for planar and axisymmetric compressible flows", Finite Elements in Analysis and Design, 46(8), pp. 613-624, 2010. https://doi.org/10.1016/j.finel.2010.03.001

[35] Ledger, P. D., Morgan, K., Peraire, J., Hassan, O., Weatherill, N. P. "The development of an hp-adaptive finite element procedure for electromagnetic scattering problems", Finite Elements in Analysis and Design, 39(8), pp. 751-764, 2003. https://doi.org/10.1016/S0168-874X(03)00057-X
[36] Badnava, H., Msekh, M. A., Etemadi, E., Rabczuk, T. "An h-adaptive thermo-mechanical phase field model for fracture", Finite Elements in Analysis and Design, 138, pp. 31-47, 2018. https://doi.org/10.1016/j.finel.2017.09.003

[37] Soghrati, S., Nagarajan, A., Liang, B. "Conforming to interface structured adaptive mesh refinement: New technique for the automated modeling of materials with complex microstructures", Finite Elements in Analysis and Design, 125, pp. 24-40, 2017. https://doi.org/10.1016/j.finel.2016.11.003

[38] Lackner, R., Mang, H. A. "Adaptive FE analysis of RC Shells. I: Theory", Journal of Engineering Mechanics, 127(12), pp. 1203$1212,2001$. https://doi.org/10.1061/(ASCE)0733-9399(2001)127:12(1203)

[39] Lackner, R., Mang, H. A. "Adaptivity in computational mechanics of concrete structures", International Journal for Numerical and Analytical Methods in Geomechanics, 25(7), pp. 711-739, 2001. https://doi.org/10.1002/nag.152

[40] Chen, S. H. "Computational Geomechanics and Hydraulic Structures", Springer Tracts in Civil Engineering, Singapore, 2019. https://doi.org/10.1007/978-981-10-8135-4

[41] Kaveh, A., Hassani, B., Shojaee, S., Tavakkoli, S. M. "Structural topology optimization using ant colony methodology", Engineering Structures, 30(9), pp. 2559-2565, 2008. https://doi.org/10.1016/j.engstruct.2008.02.012

[42] Kaveh, A., Talatahari, S. "Optimum design of skeletal structures using imperialist competitive algorithm", Computers \& Structures, 88(21-22), pp. 1220-1229, 2010. https://doi.org/10.1016/j.compstruc.2010.06.011

[43] Rahami, H., Kaveh, A., Gholipour, Y. "Sizing, geometry and topology optimization of trusses via force method and genetic algorithm", Engineering Structures, 30(9), pp. 2360-2369, 2008. https://doi.org/10.1016/j.engstruct.2008.01.012

[44] Kaveh, A., Zolghadr, A. "Magnetic charged system search for structural optimization", Periodica Polytechnica Civil Engineering, 58(3), pp. 203-216, 2014. https://doi.org/10.3311/PPci.7460

[45] Lebaal, N. "Robust low cost meta-modeling optimization algorithm based on meta-heuristic and knowledge databases approach: Application to polymer extrusion die design", Finite Elements in Analysis and Design, 162, pp. 51-66, 2019. https://doi.org/10.1016/j.finel.2019.05.004

[46] Kaveh, A. "Advances in Metaheuristic Algorithms for Optimal Design of Structures", Springer, Cham, Switzerland, 2017. https://doi.org/10.1007/978-3-319-46173-1

[47] Kaveh, A. "Applications of Metaheuristic Optimization Algorithms in Civil Engineering", Springer, Cham, Switzerland, 2017. https://doi.org/10.1007/978-3-319-48012-1

[48] Kaveh, A., Mahdavi, V. R. "Colliding bodies optimization: a novel meta-heuristic method", Computers \& Structures, 139, pp. 18-27, 2014.

https://doi.org/10.1016/j.compstruc.2014.04.005

[49] Kaveh, A., Ardalani, S. "Cost and CO2 Emission Optimization of Reinforced Concrete Frames Using Enhanced Colliding Bodies Algorithm", Asian Journal of Civil Engineering, 17(6), pp. 831858, 2016. 
[50] Kaveh, A., Moradveisi, M. "Nonlinear analysis based optimal design of double-layer grids using enhanced colliding bodies optimization method", Structural Engineering and Mechanics, 58(3), pp. 555-578, 2016.

https://doi.org/10.12989/sem.2016.58.3.555

[51] Kaveh, A., Bolandgerami, A. "Optimal design of large-scale space steel frames using cascade enhanced colliding body optimization", Structural and Multidisciplinary Optimization, 55(1), pp. $237-256,2017$

https://doi.org/10.1007/s00158-016-1494-2

[52] Kaveh, A., Sabeti, S. "Structural optimization of jacket supporting structures for offshore wind turbines using colliding bodies optimization algorithm", The Structural Design of Tall and Special Buildings, 27(13), Article number: e1494, 2018.

https://doi.org/10.1002/tal.1494
[53] Kaveh, A., Ilchi Ghazaan, M. "Enhanced colliding bodies optimization for design problems with continuous and discrete variables", Advances in Engineering Software, 77, pp. 66-75, 2014. https://doi.org/10.1016/j.advengsoft.2014.08.003

[54] Kaveh, A., Bakhshpoori, T. "Metaheuristics: Outlines, MATLAB Codes and Examples", Springer, Cham, Switzerland, 2019. https://doi.org/10.1007/978-3-030-04067-3 